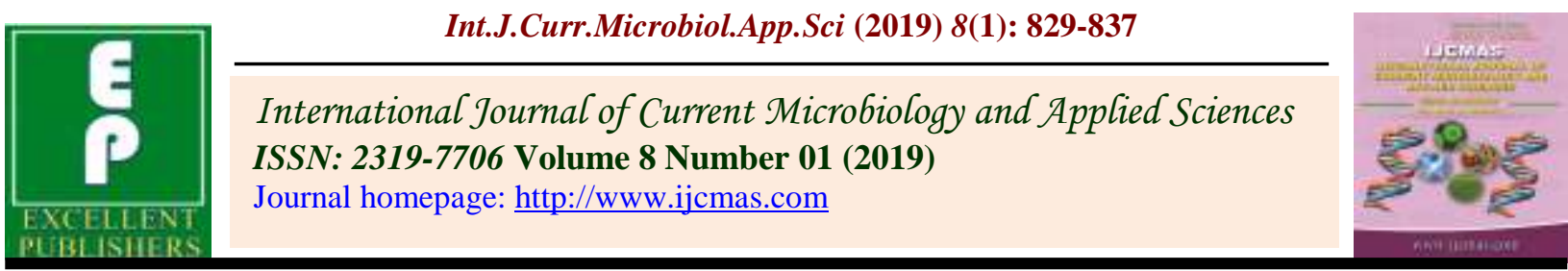

Original Research Article https://doi.org/10.20546/ijcmas.2019.801.090

\title{
Medicinal Plants Biodiversity of some Selected Villages of Zanskar Valley (Ladakh region)
}

\author{
Tsering Lamo, Tashi Stobgais, Padma Gurmet, Tsewang Dolma, Sonam Dawa*, \\ Tsering Angdus and Thinles Chosdup
}

National Research Institute for Sowa-Rigpa Leh Ladakh, Ministry of AYUSH, India

*Corresponding author

\begin{tabular}{l} 
K e y w o r d s \\
$\begin{array}{l}\text { Sowa rigpa, Amchi } \\
\text { (traditional docter), } \\
\text { Trans-Himalaya, } \\
\text { Medicinal plants, } \\
\text { Cultivation }\end{array}$ \\
\hline Article Info \\
\hline $\begin{array}{l}\text { Accepted: } \\
\text { 07 December } 2018 \\
\text { Available Online: } \\
\text { 10 January } 2019\end{array}$ \\
\hline
\end{tabular}

A B S T R A C T

The paper deals with the ethno-botanical studies of medicinal plants of some selected Zanskar valleys covering four villages Rangdum, Padum, Muney and Raru. Zanskar is one of the remote, under developed, hilly region and least populated valley of Ladakh. Because of its cold climatic condition and in accessibilities, the valley is cut off from rest of the world most of the time. Though the valley looks barren and lifeless still it represents a treasure house of diversity of plants with high medicinal, aromatic value and other useful properties like food, fodder, fuel and ritual purposes. Predominant species of these area are: Meconopsis aculeate, Rhodiola tibetica, Rheum australe, Epilobium lantifolium, Artemisia dracanculus. These medicinal plants listed below are highly used to prepare medicine for curing various diseases in Sowa rigpa. Sowa rigpa, a Tibetan system of medicine practicing by the people of trans-Himalayan region from long years ago. Raw material of indigenous species is used for preparation of medicine. People have strong belief and faith on Tibetan system of medicine therefore we need to develop it for the present and future generation. Paper enumerated 22 medicinal plants alphabetically along with detail information which belongs to different families and genera's. Conservation and cultivation of these medicinal plants are important for sustainable use because due to the unaware exploitation by locals, anthropogenic activities, overgrazing, etc may leads to the extinction of these rare species of medicinal plants. Other reason for the rarity of species may be change in climate and environmental pollution. With the rise in demand of herbal medicines globally, conservation and cultivation of medicinal plants becomes great significance for the farmer's and in turn improve their income generation.

\section{Introduction}

Ladakh, the land of high passes is located in the northern part India ( $\mathrm{J} \& \mathrm{~K}$ state), covers $70,000 \mathrm{sq} \mathrm{km}^{2}$ geographical area of the state which lies between $31^{\circ} 44^{\prime} 57^{\prime}$ '- $32^{\circ} 59^{\prime} 57^{\prime}$ 'N latitude and $76^{\circ} 46^{\prime} \quad 29^{\prime} '-8^{\circ} 41^{\prime} \quad 34^{\prime \prime} \mathrm{E}$ longitude (Hamid and Raina, 2014). Zanskar lies to the north of main Himalaya lies between $11000 \mathrm{ft}$ to $14000 \mathrm{ft}$ of altitude with huge diversity of plants. The Indian transHimalaya covers $186,000 \mathrm{~km}^{2}$ above natural tree line zone with sparsely distributed vegetation (Chaurasia and Gurmet, 2003). The 
flora of Himalaya comes under alpine and subalpine zone and due to its unique climate condition plants are sparsely distributed, dominated by herbs, shrubs, grasses and bushes. The mountain slopes and barren land display a magnificient view of flowers of the region.

The entire Ladakh is categorised into five valleys namely, Indus, Nubra, Changthang, Suru and Zanskar (Kaul, 1997). And this paper deals with ethno-botanical study of Zanskar valley, Zanskar is one of the beautiful valley of Ladakh located in west. Ethnobotany is the study of interaction between people and plant with emphasis on traditional tribal culture (Mefsin et al., 2013). The valley is separated from rest part of the Ladakh with high mountain bounding the valley from all direction and Zanskar river. As we enter the Zanskar valley, there comes a mountain pass called Penzi La with $14450 \mathrm{ft}$, after crossing this pass the different villages of Zanskar valley visible one by one. The important villages with rich medicinal plants are Rangdum, Padum, Bardan and Raru. People of Zanskar valley depend on Amchi system of medicine (a traditional healer), during earlier period it was the only health care system but later allopathic medical system came into picture after the independence of India (Chaurasia et al., 2007) (Fig. 1).

\section{The people of Zanskar}

The valley is inhabited by two tribe in Zanskar Bot tribe and Muslim tribe; mostly there is Bot tribe which are Buddhist community. Most of the people are illiterate and their main sources of income are animal rearing and agriculture. Food habit is generally both vegetarian and non vegetarian. There is a small patch of agricultural farm where they grow wheat, barley and pea. They grow vegetables in the garden for their own purpose.

\section{Climate condition of Zanskar valley}

The valley comes under one of the extreme cold and hot climate condition where summer season is very short with high radiation, low precipitation, low humidity and winter season is long with the temperature below $-27^{\circ}$ result in heavy snowfall. Therefore, due to the harsh climate condition the valley is cut off from rest of the world by the huge mountain passes and that's why people of this valley have to rely upon their own traditional method of living where people store the vegetables grown during summer season (Gurmet et al., 2000). Due to the side effect and high cost of modern medicine people are mainly depend on traditional system of medicine. The rich diversity of medicinal plants is due to peculiar temperature and type of soil of Zanskar valley.

\section{Sowa-Ripa (The Himalayan art of healing)}

Sowa-rigpa derived from Mongolian word "Am-rjay" means superior of all, commonly known as Amchi or Tibetan system of medicine and the practitioner of it is called Amchi. Since long years ago people have so much faith, trust and respect on traditional or Amchi system of medicine and this system of medicine is also very well known in Tibet, Mongolia, Bhutan, China, Nepal, Bhuriat Republic of Russia and Himalaya region of Himachal Pradesh and Sikkim in India (Wabe et al., 2011). It was the only healing method during the earlier time, later with much progress and development allopathic medicine was came. Every village have an Amchi, it takes several years to become a skillful Amchi. Earlier, it was knowledge passes from father to son now it can be practise by all. Amchis never ask for cost and services, it all depends on the people to present something beside's money like earlier time people offer wheat, barley or help the Amchi family during the time of harvesting the crops, etc (Gurmet, 2004). Rgyud-bzi, a fundamental text book of 
sowa rigpa believed to taught by Buddha outlines a vast knowledge of medicine, basic principle of health and disease, method of diagnose disease and therapeutic approaches. The basic theory of sowa rigpa is based on the principles of Jung-wa-lna (English- five elements, Sanskrit - Panch-mahabhuta) and Nespa gsum (English - three humours, Sanskrit - Tri-dosh) (Yuthog 2008). All the medicine either in powder form or capsules are made up of 3 to 40 ingredients. Stan Gyur, a Buddism text book contain various aspects of medicine and treatment of many incurable disease (Phunstog, 2006).

\section{Study area}

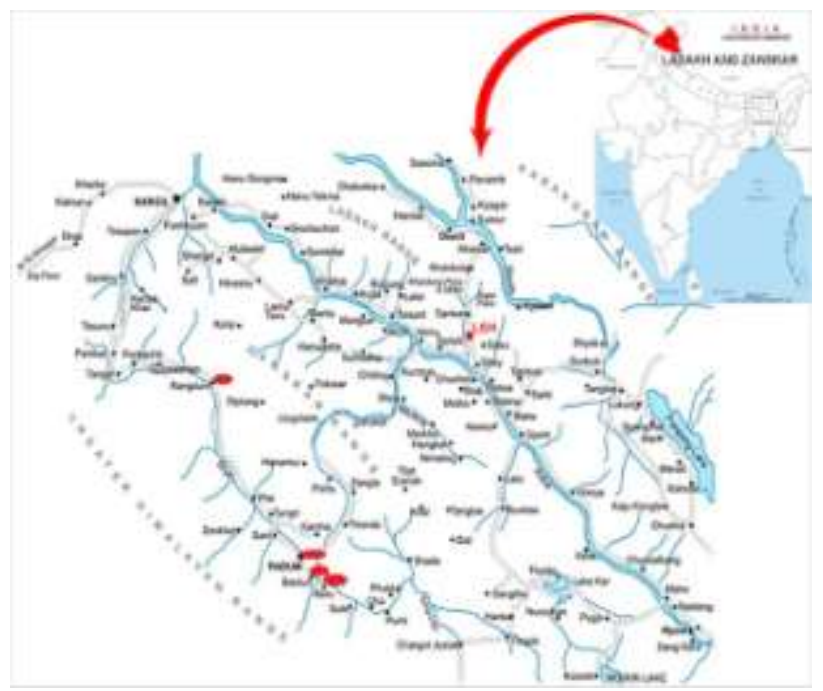

Fig.1 Map of Zanskar valley showing the study area

\section{Materials and Methods}

The survey is basically done with the aim to identify medicinal plants, to collect medicinal plants for documentation and herbarium preparation. Due to short growing season survey can carried out only during the summer season because it is very cold during winter.

The villages have been surveyed through rocky mountains, pastured land, wetlands, crossing the passes and agriculture land.
Around 60 medicinal plants were collected out of which 22 were selected for present study. Plant specimens collected from the area were dried, pressed and kept in a herbarium sheet. Herbarium were made for all the plants and kept in National Research Institute for Sowarigpa, Leh. With the help of traditional healers uses of plants was documented. The botanical plant specimens were identified with the help of flora and literary survey. The essential medicinal plants used in Sowa-rigpa were given below in alphabetical order along with botanical name, family, local name, habitat, characteristic feature and Sorig uses.

\section{Results and Discussion}

It is represented in table 1.

In conclusion since, Zanskar is one of the hotspot for medicinal plants and there is high risk of extinction of those medicinal plants due to lack of knowledge, over collection by traditional healer and researcher, unscientific exploration, uprooting for fuel, overgrazing and other activities. There are natural enemies also like pathogen, herbivores and predator (Kala et al., 2006).

Cultivation of medicinal plants can provide income for the people living in the remote area. It is therefore becomes important to conserve and cultivate those medicinal plants for future generation.

To cultivate medicinal plants, plant material should be of good quality, rich in active ingredients, pest and disease resistant and environment tolerant. Cultivation can be done either by ex-situ or in-situ method. Appropriate agro-techniques, awareness among interested farmer's, supportive government policies, protectable cultivation practise, and assured market are the key factors for successful medicinal plants cultivation. 
Table.1 Enumerations

\begin{tabular}{|c|c|c|c|c|c|c|}
\hline Botanical name & $\begin{array}{l}\text { Sowa-rigpa } \\
\text { name }\end{array}$ & Family & Habitat & Botanical feature & Part use & Sowa-rigpa uses \\
\hline $\begin{array}{l}\text { Arnebia euchroma } \\
\text { (Royle.) John. I. M } \\
\text { Johnston. }\end{array}$ & $\begin{array}{l}\text { Demok, ('bri- } \\
\text { mog) }\end{array}$ & Boraginaceae & $\begin{array}{l}\text { dry rocky } \\
\text { slopes of } \\
\text { Zanskar }\end{array}$ & $\begin{array}{l}\text { A tufted, perennial herb. Stout } \\
\text { rootstock. Stem erect, smooth, many } \\
\text { arising from rootstock. Leaves sessile, } \\
\text { alternate, pinnatisect, exstipulate, leaf } \\
\text { outline lanceolate, leaf margin ciliate, } \\
\text { leaf apex truncate and leaf base } \\
\text { cuneate. Infloresence racemose. } \\
\text { Flower blackish purple. }\end{array}$ & Root & $\begin{array}{l}\text { It treats lungs and pulmonary } \\
\text { diseases, blood disorder, nose } \\
\text { bleeding, impure blood, hair } \\
\text { losses, etc. }\end{array}$ \\
\hline $\begin{array}{l}\text { Astragalus } \\
\text { rhizanthus Royle ex } \\
\text { Benth. }\end{array}$ & (srad-ser) & Fabaceae & $\begin{array}{l}\text { On open } \\
\text { stony slopes } \\
\text { of Zanskar } \\
\text { valley }\end{array}$ & $\begin{array}{l}\text { Stemless, perennial herb, densely } \\
\text { covered with silky hairs. Leaves } \\
\text { pinnate, pressed to the ground and } \\
\text { radiating from the centre, } \\
\text { imparipinnately compound, oblong, } \\
\text { pilose beneath. Flowers bright yellow } \\
\text { in dense stalkless at the centre, } \\
\text { aggregate at the base. }\end{array}$ & $\begin{array}{l}\text { leaves, } \\
\text { stem, } \\
\text { flower and } \\
\text { fruit }\end{array}$ & $\begin{array}{l}\text { It treats fever of nerve, fever of } \\
\text { wounds, bleeding, high altitude } \\
\text { sickness and weakness, etc. }\end{array}$ \\
\hline $\begin{array}{l}\text { Artemisia } \\
\text { dracanculus Linn. } \\
\text { A.glauca ex Wild }\end{array}$ & (Tshar-bong) & Asteraceae & $\begin{array}{l}\text { on } \\
\text { wastelands } \\
\text { of Zanskar }\end{array}$ & $\begin{array}{l}\text { An erect, strong perennial herb. Stem } \\
\text { grooved, smooth. Leaves entire, } \\
\text { linear-oblong, acute. Flower heads } \\
\text { globular, short-stalk, forming long } \\
\text { axillary raceme. Involucral bracts } \\
\text { glacous with papery margin }\end{array}$ & $\begin{array}{l}\text { all parts } \\
\text { above } \\
\text { ground }\end{array}$ & $\begin{array}{l}\text { It treats pharyngitis, pulmonary } \\
\text { diseases and swelling due to hot } \\
\text { disorders etc. }\end{array}$ \\
\hline $\begin{array}{l}\text { Calvatia cyathiformis } \\
\text { (Bosc.) Morg }\end{array}$ & $\begin{array}{l}\text { (Pha-wa- } \\
\text { sGo-go) }\end{array}$ & Lycoperdaceae & grassy area & $\begin{array}{l}\text { a smooth, spherical, white at young } \\
\text { and brown at maturity. As it reach the } \\
\text { reproductive stage the exterior part } \\
\text { shrunk and the spores will disperse, } \\
\text { After the spores becomes disperse } \\
\text { there left only a leathery cup shaped } \\
\text { structure rooted at the ground. }\end{array}$ & Spores & $\begin{array}{l}\text { The powder enclosed in this } \\
\text { mushroom can be applied } \\
\text { directly on the affected part to } \\
\text { assist blood clotting and to heal } \\
\text { ruptured capillaries and wounds. } \\
\text { It is also mixed with water for } \\
\text { external application against }\end{array}$ \\
\hline
\end{tabular}




\begin{tabular}{|c|c|c|c|c|c|c|}
\hline & & & & & & $\begin{array}{l}\text { burns. It can be formulated with } \\
\text { other ingredient to treat snake } \\
\text { poison and also used in preparing } \\
\text { ink. }\end{array}$ \\
\hline $\begin{array}{l}\text { Corydalis govaniana } \\
\text { Wall. }\end{array}$ & (sKra-bZang) & Papaveraceae & $\begin{array}{l}\text { Forest area } \\
\text { and moist } \\
\text { places and } \\
\text { humid } \\
\text { condition }\end{array}$ & $\begin{array}{l}\text { stout branched, annual herb. } \\
\text { Rootstock woody often branched } \\
\text { covered with leaf bases of old leaves. } \\
\text { Leaves many, oblong, and 2- } \\
\text { pinnatisect. Flowers many, dense, } \\
\text { yellow in dense cluster. }\end{array}$ & $\begin{array}{l}\text { all parts } \\
\text { above } \\
\text { ground. }\end{array}$ & $\begin{array}{l}\text { It treats excessive impure blood, } \\
\text { fever of blood, liver and gall } \\
\text { bladder, pain due to hot } \\
\text { disorders. }\end{array}$ \\
\hline $\begin{array}{l}\text { Delphinium } \\
\text { cashmirianum Royle. }\end{array}$ & (cha-rKang) & Ranunculaceae & $\begin{array}{l}\text { Snow } \\
\text { melted } \\
\text { alpine } \\
\text { slopes }\end{array}$ & $\begin{array}{l}\text { an erect, gladrous, perennial herb. } \\
\text { Stem hispidaly hairy, branched, few } \\
\text { leaves. Leaves deeply dissected, } \\
\text { palmate with cuneate-ovate. Flower } \\
\text { bluish-purple with short spur in } \\
\text { terminal racemes, long stalk. }\end{array}$ & $\begin{array}{l}\text { all parts } \\
\text { above } \\
\text { ground. }\end{array}$ & $\begin{array}{l}\text { It treats dysentery, diarrhea with } \\
\text { bleeding, inflammation wounds, } \\
\text { lumph fluids etc. }\end{array}$ \\
\hline $\begin{array}{l}\text { Dianthus anatolicus } \\
\text { Boiss }\end{array}$ & (sukpa-rigs) & Caryophyllaceae & dry places & $\begin{array}{l}\text { A small, slender, densely tufted, } \\
\text { perennial herb. Stem } 6-10 \text { inches. } \\
\text { Leaves small, narrow, midrib and } \\
\text { margin thick. Flowers rosy. Corolla } \\
\text { toothed, blade small, broad, crenate } \\
\text { toothed. }\end{array}$ & $\begin{array}{l}\text { all parts } \\
\text { above } \\
\text { ground. }\end{array}$ & $\begin{array}{l}\text { leaves use against stomach } \\
\text { problem }\end{array}$ \\
\hline $\begin{array}{l}\text { Epilobium } \\
\text { lantifolium Linn. }\end{array}$ & $\begin{array}{l}\text { (charpan- } \\
\text { chutsi) }\end{array}$ & Onagraceae & $\begin{array}{l}\text { Damp } \\
\text { places }\end{array}$ & $\begin{array}{l}\text { An erect, leafy, perennial herb. Stem } \\
\text { branched, glabrous, often spreading. } \\
\text { Leaves narrowed above and below, } \\
\text { oblong, elliptic, pubescent. Flower } \\
\text { purplish pink in a spike like terminal } \\
\text { cluster. Calyx lobes broad lanceolate, } \\
\text { long pointed, free at base. Petals } 4, \\
\text { rounded, short stalk. Capsule long and } \\
\text { pubescent. }\end{array}$ & $\begin{array}{l}\text { Above } \\
\text { ground }\end{array}$ & Every part of this plant is edible. \\
\hline $\begin{array}{l}\text { Ephedra gerardiana } \\
\text { Wall.ex. Stapf. }\end{array}$ & $\begin{array}{l}\text { (mTshe- } \\
\text { lDum) }\end{array}$ & Ephedraceae & $\begin{array}{l}\text { stony dry } \\
\text { area of }\end{array}$ & $\begin{array}{l}\text { A low growing tufted shrub. Stem } \\
\text { erect, tufted, branched. Branches }\end{array}$ & $\begin{array}{l}\text { Fruit and } \\
\text { stem }\end{array}$ & $\begin{array}{lll}\text { It treats } & \text { various } & \text { kinds of } \\
\text { bleeding, } & \text { spleenic } & \text { diseases, }\end{array}$ \\
\hline
\end{tabular}




\begin{tabular}{|c|c|c|c|c|c|c|}
\hline & & & Zanskar & $\begin{array}{l}\text { scaly on joints. Male cones ovate, } \\
\text { solitary. Female cones solitary. Fruits } \\
\text { ovoid, red, fleshy bracts. Seed two, } \\
\text { black. }\end{array}$ & & $\begin{array}{l}\text { hepatic diseases, new and chronic } \\
\text { fever, wounds, tumuors, } \\
\text { discomfort of breathing, cough, } \\
\text { sweating, urine obstruction, } \\
\text { physical exhaustion etc. }\end{array}$ \\
\hline $\begin{array}{l}\text { Gentianella } \\
\text { moorcroftiana (Wall. } \\
\text { ex Griseb) Airy } \\
\text { Shaw }\end{array}$ & $\begin{array}{l}\text { (Spang gain } \\
\text { karpo) }\end{array}$ & Gentianaceae & $\begin{array}{l}\text { Open slopes } \\
\text { and moist } \\
\text { places. }\end{array}$ & $\begin{array}{l}\text { A slender, erect, annual herb. Stem } \\
\text { erect, branched from the base. Leaves } \\
\text { lanceolate or oblong, elliptic, } \\
\text { narrowed below. Flowers blue to } \\
\text { white, solitary in terminal or axillary } \\
\text { raceme, long pedicillate, tubular to } \\
\text { funnel shaped. }\end{array}$ & $\begin{array}{l}\text { all parts } \\
\text { above } \\
\text { ground. }\end{array}$ & antitoxin and febrifuge. \\
\hline $\begin{array}{l}\text { Meconopsis aculeate } \\
\text { Royle. }\end{array}$ & $\begin{array}{l}\text { (Tsher- } \\
\text { sNgon) }\end{array}$ & Papaveraceae & $\begin{array}{l}\text { Rare on } \\
\text { damp slopes }\end{array}$ & $\begin{array}{l}\text { delicate perennial herb. Long stem } \\
\text { with bristle hair. Leaves pinnatisect, } \\
\text { bristly hair. Flower usually blue, } \\
\text { borne on long stock. Petal usually } \\
\text { rounded to obovate. Numerous yellow } \\
\text { stamen. Capsule oblong, prickly. }\end{array}$ & $\begin{array}{l}\text { all parts } \\
\text { above } \\
\text { ground. }\end{array}$ & $\begin{array}{l}\text { It treats bone fractured, bones } \\
\text { fever, head injured, wounds and } \\
\text { strengthen bone marrow etc. }\end{array}$ \\
\hline $\begin{array}{l}\text { Melilotus officinalis } \\
\text { Linnn. }\end{array}$ & $\begin{array}{l}\text { (rGya-sPos- } \\
\text { dMan-pa) }\end{array}$ & Fabaceae & wet places. & $\begin{array}{l}\text { An erect, biennial herb. Stem slender, } \\
\text { branched, glabrous. Leaves } 3 \text { pinnate, } \\
\text { toothed, trifoliate, leaflet obovate, } \\
\text { oblong. Flowers yellow in lax raceme, } \\
\text { dense, shortly stalked bracteates. } \\
\text { Corolla yellow, odorous, usually } 3 \\
\text { times long as calyx. }\end{array}$ & $\begin{array}{l}\text { all parts } \\
\text { above } \\
\text { ground. }\end{array}$ & $\begin{array}{l}\text { It treats fever associated with } \\
\text { poison, severe bacterial diseases, } \\
\text { chronic fever, swelling, splenic } \\
\text { cramps, diphtheria, microbial } \\
\text { diseases, limbs puses etc. }\end{array}$ \\
\hline $\begin{array}{l}\text { Oxyria digyna } \\
\text { (Linn.) Hill. }\end{array}$ & (Lug-sho) & Polygonaceae & $\begin{array}{l}\text { Moist } \\
\text { places }\end{array}$ & $\begin{array}{l}\text { A fleshy acid flavoured, glabrous, } \\
\text { succulent, perennial herb. Stem } \\
\text { usually unbranched, reddish and } \\
\text { hairless. Basal leaves fleshy, } \\
\text { numerous, round to reniform, long } \\
\text { petiolated with dichotomous venation. } \\
\text { Flower minute, pink or green. }\end{array}$ & $\begin{array}{l}\text { flower and } \\
\text { stem. }\end{array}$ & $\begin{array}{l}\text { It treats wound infectious and } \\
\text { pimples etc. }\end{array}$ \\
\hline Pleurospermum & (rtsad-rgod) & Apiaceae & On moist & A stout, perennial plant. Stem hollow, & fruit & It treats all kinds of poison like \\
\hline
\end{tabular}




\begin{tabular}{|c|c|c|c|c|c|c|}
\hline $\begin{array}{l}\text { candollii (DC.) } \\
\text { Benth ex Clarke }\end{array}$ & & & slopes & $\begin{array}{l}\text { usually very thick. Base of the stem } \\
\text { covered with persistent old leaf base. } \\
\text { Leaves in basal rosette, pinnate, with } \\
\text { sheathing leaf base, leaflet broad } \\
\text { wedge shape to broad ovate, usually } 3 \\
\text { lobed and toothed. Flower white } \\
\text { borne in a solitary terminal compound } \\
\text { umbel. Fruits oblong with narrow } \\
\text { wings. }\end{array}$ & & $\begin{array}{l}\text { green poison, meat poison, } \\
\text { fragrance poison, gemstones } \\
\text { poison and transmitted poison, } \\
\text { heals all type of fever, maintain } \\
\text { body, constipation, pain and } \\
\text { amenorrhoea, etc. }\end{array}$ \\
\hline $\begin{array}{l}\text { Rhodiola tibetica } \\
\text { (Hk.f. \& T.) Fu }\end{array}$ & $\begin{array}{l}\text { (Sro-lo-dmar- } \\
\text { po) }\end{array}$ & Crassulaceae & $\begin{array}{l}\text { common on } \\
\text { moist alpine } \\
\text { places }\end{array}$ & $\begin{array}{l}\text { An erect, perennial herb. Stem fleshy, } \\
\text { pubescent, green. Leaves fleshy, } \\
\text { lanceolate, green. Flower reddish, } \\
\text { stalkless, bractless, in a dense domed } \\
\text { cluster }\end{array}$ & $\begin{array}{l}\text { All parts } \\
\text { above } \\
\text { ground }\end{array}$ & $\begin{array}{l}\text { It heals lungs disorder, fever of } \\
\text { the lungs, asthma, any type of } \\
\text { mouth-disorders, infectious } \\
\text { cough and especially for body } \\
\text { tonic etc. }\end{array}$ \\
\hline $\begin{array}{l}\text { Rheum australe D. } \\
\text { Don Syn. (R.emodi } \\
\text { Wall. ex Meisser) }\end{array}$ & (Chhu-rTsa) & Polygonaceae & $\begin{array}{l}\text { Open } \\
\text { slopes, } \\
\text { alpine } \\
\text { grassland }\end{array}$ & $\begin{array}{l}\text { Perennial with stout stem. Leaves } \\
\text { rounded to broadly ovate blade, basal } \\
\text { leaves are very large, reddish brown } \\
\text { at maturity. Rootstock stout. }\end{array}$ & $\begin{array}{l}\text { root, } \\
\text { leaves and } \\
\text { trunk also } \\
\text { use } \\
\text { sometime }\end{array}$ & $\begin{array}{l}\text { It treats bone fracture, } \\
\text { indigestion, bloated stomach, } \\
\text { cancers, severe bacterial disease } \\
\text { tumours, dermatological } \\
\text { disorders, wounds, internal } \\
\text { wounds, fever of gall bladder and } \\
\text { amenorrhoea etc. Also used for } \\
\text { colouring wool and as fixative. } \\
\text { Petioles are used as anthelmintic, } \\
\text { and ingastritis and swellings. } \\
\text { Also used as pickle. }\end{array}$ \\
\hline $\begin{array}{l}\text { Saussurea obvallata } \\
\text { (DC) Edgew }\end{array}$ & $\begin{array}{l}\text { (gZah-dug- } \\
\text { nag-po) }\end{array}$ & Asteraceae & Grassy area. & $\begin{array}{l}\text { An erect, robust, perennial herb with } \\
\text { stout stem. Leaves amplexicaul, } \\
\text { toothed, lower and upper leaf sessile, } \\
\text { arcuate venation. Flower head purple } \\
\text { in dense umble like cluster } \\
\text { surrounded by large pale yellow boat } \\
\text { shaped pappery bracts. }\end{array}$ & $\begin{array}{l}\text { All parts is } \\
\text { used }\end{array}$ & $\begin{array}{l}\text { It treats evil spirits diseases, } \\
\text { planetary diseases, wound and } \\
\text { relief pain etc. }\end{array}$ \\
\hline ewersii & (tsan-rigs) & Crassulaceae & moist & A small, succulent, glabrous, & part & Leaves are used as a salad and for \\
\hline
\end{tabular}




\begin{tabular}{|c|c|c|c|c|c|c|}
\hline Ledeb. & & & slopes. & $\begin{array}{l}\text { perennial herb. Stem usually branched } \\
\text { at the base, reddish. Leaves mostly } \\
\text { opposite, ovate to round, younger } \\
\text { leaves reddish and older leaves } \\
\text { greenish. Flower small purple borne } \\
\text { in cluster at the top. }\end{array}$ & $\begin{array}{l}\text { above } \\
\text { ground are } \\
\text { used. }\end{array}$ & making sauce. \\
\hline $\begin{array}{l}\text { Tanacetum tibeticum } \\
\text { Hk.f \& T. Ex Clarke }\end{array}$ & $\begin{array}{l}\text { (khanchung- } \\
\text { srego) }\end{array}$ & Asteraceae & $\begin{array}{l}\text { Open slopes } \\
\text { and } \\
\text { wasteland }\end{array}$ & $\begin{array}{l}\text { Stem many arising from woody } \\
\text { rootstock. Leaves much dissected. } \\
\text { Flower head yellow, large, rounded, } \\
\text { forming terminal corymbs. }\end{array}$ & $\begin{array}{l}\text { all part } \\
\text { above } \\
\text { ground are } \\
\text { used. }\end{array}$ & $\begin{array}{l}\text { used as an antiseptic, against } \\
\text { swelling gums }\end{array}$ \\
\hline $\begin{array}{l}\text { Thlaspi alpestre } \\
\text { Linn.ex.Hk.f.\& } \\
\text { T.Anders }\end{array}$ & (dayga) & Brassicaceaea & Rocky area & $\begin{array}{l}\text { A small perennial tufted herb. Stem } \\
\text { erect, glabrous, simple or branched. } \\
\text { Leaves orbicular, cauline leaves } \\
\text { obovate, auricle. Flower large borne } \\
\text { in a raceme. Pods triangular, winged. }\end{array}$ & $\begin{array}{l}\text { fruit, } \\
\text { leaves, } \\
\text { flower }\end{array}$ & $\begin{array}{l}\text { used against inflammation of } \\
\text { lungs and kidney, seminal and } \\
\text { vaginal discharges, kidney } \\
\text { problem, pus in lungs, and } \\
\text { appendicitis. }\end{array}$ \\
\hline
\end{tabular}


DIHAR (Defence Institute of High Altitude Research) has cultivated the medicinal plants by vegetative propagation and they also conducted workshops and field demonstration on conservation practise of medicinal plants. NGO's, stakeholder's, scientist and government organization should work collaboratively to conserve the valuable medicinal plants for the sustainable use.

In spite of advancement in modern medicine people still rely on traditional healing practise of herbal based medicine for health care as old as human civilization. Traditional medicines are effective, safe, inexpensive and culturally acceptable while modern medicines are expensive and show side effect. About $80 \%$ of world population are based on herbal products for primary health care but due to lack of interest in younger generation this traditional knowledge has been started declining. Hence it becomes important for all of us to conserve traditional knowledge

\section{Acknowledgement}

Authors are thankful to National Research Institute for Sowa-Rigpa (CCRAS), Ministry of AYUSH for support to make it successful.

\section{References}

Chaurasia, O.P. and Gurmet, P. 2003. A Checklist on medicinal and aromatic plants of trans-himalaya cold desert.

Chaurasia, O.P., Ahmed, Z. and Ballabh, B. 2007. Ethnobotany and plants of transHimalaya.

Gurmet, P. 2004. Sowa-rigpa; Himalayan art of healing. Indian Journal of
Traditional Knowledge 3(2): 212-218.

Gurmet, P. Chaurasia, O.P., Brahma and. Attery, D.P. 2000. Medico-Botanical Survey of Sapi valley of Kargil (Ladakh Himalayas). Bulletin of Medico-Ethno-Botanical Research. XXI: $1-10$.

Hamid, A. and Raina, A.K. 2014. Ethnobotanical uses of Plants in and around Kanji Wildlife Sanctuary, North Himalaya. International Journal of Science and Research 3(11): 538-545.

Kala, C.P., Dhyani, P.P. and Sajwan, B.S. 2006. Developing the medicinal plants sector in northern India: challenges and opportunities. Journal of Ethnobiology and Ethnomedicine 2(32):1-15.

Kaul, M.K. 1997. Medicinal plants of Kashmir and Ladakh.

Mesfin, K., Tekle, G. and Tesfay, T. 2013. Ethnobotanical study of traditional medicinal plants used by indigenous people of Gemad district, Northern Ethiopia. Journal of Medicinal Plants Studies 1(4): 32-37.

Phuntsog: Ancient Materia Medica: Sowa rigpa (Tibetan Science of healing); 2006.

Wabe, N.T., Mohammed, A.M. and Raju, N.J. 2011. Ethnobotanical survey of medicinal plants in the Southeast Ethiopia used in traditional medicine. Spatula DD. 1(3):153-158.

Yuthog Yontan Gonpo. 2008. The basic tantra and the explanatory tantra from the secret quintessential instruction on the eight branches of the Ambrosia essence tantra.

\section{How to cite this article:}

Tsering Lamo, Tashi Stobgais, Padma Gurmet, Tsewang Dolma, Sonam Dawa, Tsering Angdus and Thinles Chosdup. 2019. Medicinal Plants Biodiversity of some Selected Villages of Zanskar Valley (Ladakh region). Int.J.Curr.Microbiol.App.Sci. 8(01): 829-837. doi: https://doi.org/10.20546/ijcmas.2019.801.090 